\title{
STRANGE PERMUTATION REPRESENTATIONS OF FREE GROUPS
}

\author{
MEENAXI BHATTACHARJEE and DUGALD MACPHERSON
}

(Received 18 November 1999; revised 21 March 2002)

Communicated by R. B. Howlett

\begin{abstract}
Certain permutation representations of free groups are constructed by finite approximation. The first is a construction of a cofinitary group with special properties, answering a question of Tim Wall published by Cameron. The second yields, via a method of Kepert and Willis, a totally disconnected locally compact group which is compactly generated and uniscalar but has no compact open normal subgroup. Finally, an oligomorphic group of automorphisms of the random graph is built, all of whose non-trivial subgroups have just finitely many orbits.
\end{abstract}

2000 Mathematics subject classification: primary $20 \mathrm{~B} 07$.

\section{Introduction}

In this paper we give three constructions of faithful permutation representations, with peculiar properties, of free groups. We collect them in the same paper more because the methods are similar than because the topics are. In each case, we define the permutation representation by finite approximation, expressing the generators as unions of finite partial functions. It is not important that the groups acting are free: indeed, by a theorem of Dixon [3], the set of pairs of permutations which generate a free group is comeagre in the natural topological space on pairs of permutations of $\mathbb{N}$ (the product topology from the usual topology on $\operatorname{Sym}(\mathbb{N})$ ), and it remains a challenge to build examples like those below which are not free.

Our permutation groups will always act on a countable set $\Omega:=\left\{\xi_{i}: i \in \mathbb{N}\right\}$. We use lower case Greek letters for elements of $\Omega$, upper case Greek letters for subsets of $\Omega$, and lower case Roman letters for group elements (except that we allow variables

(C) 2003 Australian Mathematical Society $1446-7887 / 03 \$ A 2.00+0.00$ 
$x, y$ to range through group elements or $\Omega$ ). Permutations are written on the right of their arguments.

Our first theorem, proved in Section 2, concerns cofinitary permutation groups. Recall that a permutation group $G$ on $\Omega$ is cofinitary (Cameron [2]) if every nonidentity element has just finitely many fixed points. Our theorem answers a question of Wall [2, Section 10], posed as a test of the construction methods available for cofinitary groups.

THEOREM 1.1. There is a cofinitary permutation group $G$ on the countably infinite set $\Omega$ such that $G$ is freely generated by $\left\{f_{i}: i \in \mathbb{N}\right\}$ and for each $i \in \mathbb{N}$

(a) $f_{i}$ fixes $\xi_{j}$ for $0 \leq j<i$ and acts as a single cycle on $\Omega \backslash\left\{\xi_{j}: j<i\right\}$,

(b) the group $\left\langle f_{0}, \ldots, f_{i}\right\rangle$ is not $(i+2)$-transitive.

It is evident that by condition (a), $\left\langle f_{0}, \ldots f_{i}\right\rangle$ is $(i+1)$-transitive for each $i$.

In Section 3 we construct a permutation group which provides an answer to a question of George Willis, in his work on scale functions for totally disconnected groups.

THEOREM 1.2. The free group $F_{2}=\langle f, g\rangle$ has a faithful transitive action on a countable set $\Omega$ such that the following hold, where $\Omega=\Gamma \cup \Delta$ is a partition of $\Omega$ into two infinite sets:

(a) each cycle of each element of $F_{2}$ is finite;

(b) for each $x \in F_{2}$, the symmetric difference $\Gamma \Delta \Gamma x$ is finite.

It follows that for each $x \in F_{2}$ there is $\Lambda \subset \Omega$ such that $\Lambda \triangle \Gamma$ is finite and $\Lambda x=\Lambda$, but (by transitivity) there is no $G$-invariant set $\Lambda \subset \Omega$ with $\Lambda \triangle \Gamma$ finite.

The context of this construction is as follows (see [9] or [6] for background). If $G$ is any totally disconnected locally compact group and $x \in G$, then there is a compact open subgroup $U$ of $G$ so that the following hold, where $U_{+}:=\bigcap\left(x^{n} U x^{-n}: n \in \mathbb{N}\right)$ and $U_{-}:=\bigcap\left(x^{-n} U x^{n}: n \in \mathbb{N}\right)$.

(1) $U=U_{+} U_{-}$.

(2) $\bigcup\left(x^{n} U_{+} x^{-n}: n \in \mathbb{N}\right)$ and $\bigcup\left(x^{-n} U_{-} x^{n}: n \in \mathbb{N}\right)$ are both closed subgroups of $G$.

The index function $s(x)=\left|x U_{+} x^{-1}: U_{+}\right|$, the scale function of $G$, is independent of the choice of $U$, and is a continuous function $s: G \rightarrow \mathbb{N}$ such that $s(x)=1=$ $s\left(x^{-1}\right)$ if and only if $x$ normalises some compact open subgroup of $G$. The group $G$ is called uniscalar if $s$ takes value 1 everywhere. Clearly if $G$ has a compact open normal subgroup then $G$ is uniscalar, and the converse is known to be false (see [10] for references). However, it was not previously known if there was a totally disconnected locally compact compactly generated uniscalar group with no compact 
open normal subgroup, but in [6] Kepert and Willis show that such an example can be obtained from the group constructed in Theorem 1.2. For let $K$ be a finite group, and let $H:=\sum_{\Delta} K \times \prod_{\Gamma} K$. Let $F_{2}$ act on $\Omega$ as in Theorem 1.2. Then $F_{2}$ acts on $H$ via its action on the indices, and the semidirect product $G:=H \times F_{2}$ will be a totally disconnected locally compact compactly generated uniscalar group with no compact open normal subgroup. As commented at the end of [6], for each $g \in F_{2}$ the group $G$ even has a basis of neighbourhoods of the identity consisting of compact open subgroups normalised by $g$. Possible variations on the construction are discussed at the end of Section 3.

We turn in Section 4 to ZTF groups. A permutation group on an infinite set is said to be ZTF 'Zimmer torsion-free' if each non-identity element has just finitely many cycles (so each non-trivial subgroup has finitely many orbits). R. Zimmer raised questions about the structure of such groups, in connection with ergodic theory. An easy example of a ZTF group is the infinite cyclic group acting regularly, and at the other extreme, the free group on 2-generators was shown in [7] to have a faithful ZTF action. These examples are in a sense typical, for by a result of Neumann [7, Lemma 3.3], centralisers in a ZTF group must be cyclic-by-finite. A critical question is whether there exists a highly implausible Frobenius group, that is, a Frobenius ZTF group in which point stabilisers are infinite cyclic. Recall that a permutation group on a countably infinite set is oligomorphic [1] if it has finitely many orbits on $k$-sets for all $k>0$. Neumann [7, Proposition 3.6] showed that any non-trivial ZTF group which is not oligomorphic or regular has a subgroup with a faithful highly implausible Frobenius action on some (possibly different) set. It is not known whether there is any highly implausible Frobenius group, but it is easy to see that such a group cannot be free. We remark that by [8] and [5], there is no 2-transitive permutation group whose one-point stabilisers are infinite cyclic.

The ZTF group constructed in [7] may well be highly transitive, that is, $k$-transitive for all $k>0$, and certainly the construction there can be modified to yield a highly transitive group. It is more interesting (and relevant to the existence of highly implausible ZTF groups) to consider non-highly transitive ZTF groups. As pointed out by Peter Neumann, if $(G, \Omega)$ is the permutation group built in [7], then $G$ has a 'diagonal' action on the disjoint union of two copies of $\Omega$ which is oligomorphic, ZTF, but not transitive. However, it is not so clear how to obtain a primitive but not highly transitive ZTF group. Below, we build such a group acting on the random graph (defined at the end of the section).

THEOREM 1.3. Let $(\Omega, \sim)$ be the random graph (so $\sim$ is a binary irreflexive symmetric relation on the domain $\Omega$ ), and let $\Omega:=\left\{\xi_{i}: i \in \mathbb{N}\right\}$. Then there are $f, g \in \operatorname{Aut}(\Omega, \sim)$ such that

(a) $f, g$ generate a free subgroup of $\operatorname{Aut}(\Omega, \sim)$, 
(b) $f$ has a single cycle on $\Omega$, which is infinite,

(c) $g$ fixes $\xi_{0}$ and has two cycles on $\Omega \backslash\left\{\xi_{0}\right\}$,

(d) the group $(f, g)$ is a primitive oligomorphic ZTF group.

We remark that since $\langle f, g\rangle$ is transitive on vertices, edges, and non-edges, by the primitivity criterion of Higman [4] it acts primitively on $\Omega$. By the remarks above, since $F_{2}$ is free but does not act regularly, the action is oligomorphic. It seems likely that the proof could be modified to ensure that $\langle f, g\rangle$ is also a dense subgroup of $\operatorname{Aut}(\Omega, \sim)$, that is, has the same orbits on finite ordered sets as the whole automorphism group. The proof is rather involved, but it suggests that many structures which are homogeneous (in the sense defined below) admit large ZTF groups of automorphisms. Observe though that $\operatorname{Aut}(\mathbf{Q},<)$ has no non-trivial ZTF subgroup. Furthermore, if $G$ is any oligomorphic group acting on a set $\Omega$ such that the pointwise stabiliser in $G$ of a finite subset of $\Omega$ preserves some partial ordering on $\Omega$ with an infinite chain, then the action of $G$ on $\Omega$ cannot be ZTF.

The method of proof of Theorems 1.1-1.3 is to build a permutation group generated freely by $\left\{f_{i}: i \in I\right\}$, by approximating each permutation $f_{i}$ by a chain of finite partial functions. In Section $2, I=\mathbb{N}$, and in Section 3 and Section $4, I=\{0,1\}$, with $f:=f_{0}$ and $g:=f_{1}$. We denote by $f_{i}^{(k)}$ the partial function on $\Omega$ constructed after $k$ steps, so $f_{i}:=\bigcup\left(f_{i}^{(k)}: k \in \mathbb{N}\right.$ ) (so we regard each partial function as a set of ordered pairs). If $w$ is a word in the $f_{i}$, then $w^{(k)}$ is the partial function on $\Omega$ obtained by composing the $f_{i}^{(k)}$. A partial $w^{(k)}$-cycle is a maximal sequence $\gamma_{0}, \ldots, \gamma_{t}$ from $\Omega$ (denoted $\left.\left(\ldots, \gamma_{0}, \ldots, \gamma_{t}, \ldots\right)\right)$ such that $\gamma_{0}\left(w^{(k)}\right)^{t}$ is defined and equals $\gamma_{t}$. We use the word cycle for partial cycle, and complete cycle to refer to a cycle as above where $\gamma_{t} w^{(k)}=\gamma_{0}$. A $w^{(k)}$-chain is a sequence $\left(\delta_{0}, \ldots, \delta_{t}, i, w\right) \in \Omega$ such that for some subword $u_{1} \cdots u_{f}$ of a power of $w$ (with $u_{1}$ the $i^{\text {th }}$ symbol of $w$, and with $\left.u_{1}, \ldots, u_{t} \in\left\{f, g, f^{-1}, g^{-1}\right\}\right)$, we have $\delta_{0} u_{1}^{(k)} \cdots u_{j}^{(k)}=\delta_{j}$ for each $j=1, \ldots, t$. In practice, we refer to the $w$-chain $\left(\delta_{1}, \ldots, \delta_{t}\right)$ and drop the final entries $i, w$, but formally, two $w$-chains are equal if they agree in all entries, including the final ones. A maximal $w^{(k)}$-chain is a $w^{(k)}$-chain which is not a proper subsequence of any other $w^{(k)}$-chain. The length of a maximal $w^{(k)}$-chain $\left(\delta_{0}, \ldots, \delta_{t}\right)$ is $t$. At step $k$, a new point is some $\delta \in \Omega$ such that $\delta \notin\left\{\xi_{0}, \ldots, \xi_{k}\right\}$ and such that $\delta \notin \operatorname{dom}\left(f_{i}^{(k-1)}\right) \cup \operatorname{ran}\left(f_{i}^{(k-1)}\right)$ for all $i \in I$. We often regard partial permutations as sets of ordered pairs, and we use the notation $\langle\alpha, \beta\rangle$ for ordered pairs.

A relational structure $M$ is homogeneous if its domain is countably infinite and any isomorphism between finite substructures of $M$ extends to an automorphism of $M$. The standard method of construction of homogeneous structures is Fraïssé's amalgamation theorem. The random graph, is a well-known example of a homogeneous structure. It is up to isomorphism the unique countably infinite graph $\Gamma$ satisfying the following 'extension property': for any two finite disjoint sets $U, V$ of vertices, there is a vertex adjacent to everything in $U$ and to nothing in $V$. The homogeneous 
structure constructed in Section 2, though over an infinite language, has a similar characterisation. See [1] for more on homogeneous structures, Fraïssé amalgamation, and the random graph.

\section{Proof of Theorem 1.1}

The group $G$ will be a group of automorphisms of a countable homogeneous relational structure $\Omega^{*}$ which we first construct. Let $L$ be a first order language, with, for each $n \in \mathbb{N}$, a single relation symbol $R_{n+2}$ of arity $n+2$. Let $\mathscr{C}$ be the class of all finite $L$-structures in which, for each $n \geq 0$, whenever $R\left(x_{1}, \ldots, x_{n+2}\right)$ holds, we have that (a) all the $x_{i}$ are distinct, and (b) $R\left(x_{1 g}, \ldots, x_{(n+2) g}\right)$ for each $g$ in the symmetric group $S_{n+2}$. It is routine to check that $\mathscr{C}$ is an amalgamation class, so there is a unique countable homogeneous $L$-structure $\Omega^{*}$ whose finite substructures are up to isomorphism precisely the members of $\mathscr{C}$. Let $\Omega$ denote the domain of $\Omega^{*}$, and for each $i>1$ let $\Omega_{i}^{*}$ be the reduct of $\Omega^{*}$ to the language containing only the relations $R_{j}$ for $j \geq i$ (so $\Omega_{2}^{*}=\Omega^{*}$ ). Put $\Omega=\left\{\xi_{i}: i \in \mathbb{N}\right\}$.

We build the permutations $f_{i}$ so that for each $i \in \mathbb{N}$,

(i) $f_{i}$ fixes $\xi_{j}$ for all $j<i$, and acts as a single infinite cycle on $\left\{\xi_{j}: j \geq i\right\}$, and

(ii) $f_{i} \in \operatorname{Aut}\left(\Omega_{i+2}^{*}\right)$.

Since some but not all ordered $(i+2)$-sets in $\Omega_{i+2}^{*}$ satisfy $R_{i+2}$, the group $\left\langle f_{0}, \ldots, f_{i}\right\rangle$ will not be $(i+2)$-transitive.

We construct the permutations in $\omega$ many steps, arranging that for each word in the $f_{i}, f_{i}^{-1}$, after a certain stage it acquires no new fixed points. The group $G:=\left\langle f_{i}: i \in \mathbb{N}\right\rangle$ must then be cofinitary. Each $f_{i}$ is constructed as a union of a chain of finite approximations $\left(f_{i}^{(j)}: j \geq i\right)$, where $f_{i}^{(j)}$ is the approximation of $f_{i}$ constructed after $j$ steps.

Let $W:=\left\{w_{i}: i \in \mathbb{N}\right\}$ be the set of cyclically reduced words in the $f_{i}$ and $f_{i}^{-1}$. To ensure that $G$ is cofinitary, it suffices to arrange that each element of $W$ induces a permutation of $\Omega$ with just finitely many fixed points. This ensures also that $G$ is freely generated by the $f_{i}$.

At step 0 , we put $f_{0}^{(0)}=\left(\ldots \xi_{1}, \xi_{0}, \xi_{i} \ldots\right)$, where $i \in \mathbb{N} \backslash\{0,1\}$ is least such that $R_{2}\left(\xi_{1}, \xi_{0}\right) \leftrightarrow R_{2}\left(\xi_{0}, \xi_{i}\right)$. This notation means that $\left(\ldots \xi_{1}, \xi_{0}, \xi_{i} \ldots\right)$ is a partial cycle of $f_{0}^{(0)}$, so $\xi_{1} f_{0}^{(0)}=\xi_{0}$ and $\xi_{0} f_{0}^{(0)}=\xi_{i}$, with $f_{0}^{(0)}$ not defined elsewhere.

Before the $n^{\text {th }}$ step, we will have defined $f_{j}^{(n-1)}$ for all $j<n$. Here, $f_{j}^{(n-1)}$ fixes $\xi_{k}$ for $k<j$ and has exactly one other finite partial cycle, which is incomplete and of length greater than one, and $\xi_{k} \in \operatorname{dom}\left(f_{j}^{(n-1)}\right) \cap \operatorname{ran}\left(f_{j}^{(n-1)}\right)$ for all $k \leq n-1$. This last condition guarantees that the $f_{j}$ will be defined everywhere and surjective.

At the $n^{\text {th }}$ step, we ensure that $\xi_{n} \in \operatorname{dom}\left(f_{j}^{(n)}\right) \cap \operatorname{ran}\left(f_{j}^{(n)}\right)$ for $j \leq n$, and that $f_{n}^{(n)}$ fixes $\xi_{0}, \ldots, \xi_{n-1}$. Our procedure to put $\xi_{n}$ into the domain and range of $f_{i}^{(n)}$ 
is as follows (we do this for each $i \leq n$ ). If $\xi_{n} \in \operatorname{dom}\left(f_{i}^{(n-1)}\right) \cap \operatorname{ran}\left(f_{i}^{(n-1)}\right)$, put $f_{i}^{(n)}:=f_{i}^{(n-1)}$. If $\xi_{n} \in \operatorname{dom}\left(f_{i}^{(n-1)}\right) \backslash \operatorname{ran}\left(f_{i}^{(n-1)}\right)$, choose a 'new' point $\delta \in \Omega$ ('new' as defined in the end of Section 1) and put $f_{i}^{(n)}:=f_{i}^{(n-1)} \cup\left\{\left\langle\delta, \xi_{n}\right\rangle\right\}$ (so $\delta f_{i}^{(n)}=\xi_{n}$ ). We also assume that a 'new' point for $f_{i}^{(n)}$ cannot be new for any $f_{j}^{(n)}$ where $j \in\{0, \ldots, n\} \backslash\{i\}$. The restriction on $\delta$ is that $f_{i}^{(n)}$ preserves the relations $R_{j}$ for $j \geq i+2$. Since the $R_{j}$ only hold on tuples of distinct elements, and $\operatorname{dom}\left(f_{i}^{(n)}\right)$ is finite, only finitely many $R_{j}$ need be considered (of arity at most $\left|\operatorname{dom}\left(f_{i}^{(n)}\right)\right|-1$ ), and so by the homogeneity of $\Omega^{*}$ there are infinitely many possibilities for $\delta$. Similarly, if $\xi_{n} \in \operatorname{ran}\left(f_{i}^{(n-1)}\right) \backslash \operatorname{dom}\left(f_{i}^{(n-1)}\right)$, then choose new $\delta \in \Omega$ as above and put $f_{i}^{(n)}:=$ $f_{i}^{(n-1)} \cup\left\{\left\langle\xi_{n}, \delta\right\rangle\right\}$. Also, to put $\xi_{n}$ into the domain and range of $f_{n}^{(n)}$, just choose suitable distinct new $\delta, \epsilon$ and put $f_{n}^{(n)}:=\left(\xi_{0}\right) \ldots\left(\xi_{n-1}\right)\left(\ldots, \delta, \xi_{n}, \epsilon, \ldots\right)$.

We must also consider the case when $\xi_{n} \notin \operatorname{dom}\left(f_{i}^{(n-1)}\right) \cup \operatorname{ran}\left(f_{i}^{(n-1)}\right)$, and $i<n$. Suppose that the non-trivial partial cycle of $f_{i}^{(n-1)}$ is $\left(\ldots, \alpha_{1}, \ldots, \alpha_{r}, \ldots\right)$. Choose a new point $\delta$ and then a set of distinct new points $B_{i}=\left\{\beta_{1}, \ldots, \beta_{s}\right\}$ (with $\delta \notin B_{i}$ ) where $s=\max \{r-1, n\}$, and put

$$
f_{i}^{(n)}:=\left(\xi_{0}\right) \ldots\left(\xi_{i-1}\right)\left(\ldots, \alpha_{1}, \ldots, \alpha_{r}, \beta_{1}, \ldots, \beta_{s}, \xi_{n}, \delta, \ldots\right) .
$$

The choice of $\delta$ is easy, much as in the last paragraph. The choice of the $\beta_{i}$ however needs some care, to ensure that $f_{i}^{(n)}$ preserves $R_{j}$ for $j \geq i+2$. We can ignore the fixed points $\xi_{0}, \ldots, \xi_{i-1}$, since each relation $R_{j}$ and subset of size $k$ of $\left\{\xi_{0}, \ldots, \xi_{i-1}\right\}$ determines a new relation of arity $j-k \geq i+2-k \geq 2$ on $\Omega \backslash\left\{\xi_{0}, \ldots, \xi_{i-1}\right\}$ which must be preserved by $f_{i}$, and there are finitely many of these 'new' relations (we only need to consider relations of arity less than $r+s+2$, the length of the non-trivial cycle of $f_{i}^{(n)}$ ). We have two kinds of conditions required for the $\beta_{j}$ and $\delta$. First, if one of the relations holds of a tuple from $\left\{\alpha_{1}, \ldots, \alpha_{r}\right\}$ then it must hold for any translates under $f_{i}^{(n)}$ which involve the $\beta_{i}$. Conditions of this sort have 'span' at most $r-1$, in the sense that they involve points at most $r-1$ apart in the cycle of $f_{i}^{(n)}$. Second, if a relation holds of a tuple involving $\xi_{n}$ and some of $\left\{\alpha_{1}, \ldots, \alpha_{r}\right\}$, then translates of this under $f_{i}^{(n)}$ impose conditions on the $\beta_{i}$ and $\delta$. Conditions of this second sort have span at least $s+1 \geq r$, so there is no clash between conditions of the two sorts. Thus, using the homogeneity of $\Omega^{*}$ the elements of $B_{i}$ can be found. The sets $B_{i}$ (for $i<n$ ) are all chosen to be disjoint.

It remains to verify that in this construction, each word $w_{i}$ has finitely many fixed points. Consider a word $w \in W$. As usual let $w^{(n)}$ denote the word obtained from $w$ by replacing, for each $i \in \mathbb{N}$, any occurrence of $f_{i}$ or $f_{i}^{-1}$ by $f_{i}^{(n)}$ or $\left(f_{i}^{(n)}\right)^{-1}$ respectively. Suppose that at step $n, w$ acquires a fixed point, that is, there is $\epsilon \in M$ such that $\epsilon w^{(n-1)}$ is undefined but $\epsilon w^{(n)}=\epsilon$. We shall show that either $\ell(w) \geq s$ (so $\ell(w) \geq n)$, or $f_{n}$ occurs in $w$. It follows that there is some step $t$ such that after step $t, w$ acquires no new fixed points. Since $w^{(t)}$ has just finite domain, the word $w$ has just finitely many fixed points, as required. 
We may suppose that $f_{n}$ does not occur in $w$. Step $n$ really consists of $n+1$ substeps (one for each of $f_{0}^{(n)}, \ldots, f_{n}^{(n)}$ ), and for convenience we shall suppose that $w^{(n)}$ becomes defined at $\epsilon$ at the $0^{\text {th }}$ substep, when we put $\xi_{n}$ into the domain and range of $f_{0}^{(n)}$. (The arguments for $f_{0}, \ldots, f_{n-1}$ are similar, and by the above assumption, we can ignore the substep when $f_{n}^{(n)}$ is defined as this cannot introduce a fixed point for $w$.) We shall suppose that $\xi_{n} \notin \operatorname{dom}\left(f_{0}^{(n-1)}\right) \cup \operatorname{ran}\left(f_{0}^{(n-1)}\right)$, this being the hardest case. So $f_{0}^{(n-1)}=\left(\ldots, \alpha_{1}, \ldots, \alpha_{r}, \ldots\right)$, and $f_{0}^{(n)}=\left(\ldots, \alpha_{1}, \ldots, \alpha_{r}, \beta_{1}, \ldots, \beta_{s}, \xi_{n}, \delta, \ldots\right)$, where $s=\max \{r-1, n\}$.

Clearly $f_{0}$ or $f_{0}^{-1}$ occurs in $w$. Write $w=u_{1} \cdots u_{t}$ and $w^{(n)}=u_{1}^{(n)} \cdots u_{t}^{(n)}$, where $u_{i} \in\left\{f_{j}, f_{j}^{-1}: j \in \mathbb{N}\right\}$ and $u_{i}^{(n)}$ is the approximation of $u_{i}$ after $n$ steps. There is $j \leq t$ such that $u_{1}^{(n-1)} \cdots u_{j-1}^{(n-1)}$ is defined at $\epsilon$ but $u_{1}^{(n-1)} \cdots u_{j}^{(n-1)}$ is undefined at $\epsilon$. This means that $u_{j}$ is $f_{0}$ or $f_{0}^{-1}$ and $\epsilon^{\prime}$, the image of $\epsilon$ under $u_{1}^{(n-1)} \cdots u_{j-1}^{(n-1)}$, is in $\left\{\alpha_{r}, \beta_{1}, \ldots, \beta_{s}, \xi_{n}\right\}$ (if $u_{j}=f_{0}$ ) or in $\left\{\beta_{1}, \ldots, \beta_{s}, \xi_{n}, \delta\right\}$ (if $u_{j}=f_{0}^{-1}$ ).

In the first case, when $u_{j}=f_{0}$, there are three possibilities.

(i) $\epsilon^{\prime}=\xi_{n}$;

(ii) $\epsilon^{\prime}=\alpha_{r}$;

(iii) $\epsilon^{\prime} \in\left\{\beta_{1}, \ldots, \beta_{s}\right\}$ (in which case $j=1$ as the $\beta_{i}$ are new).

As $\xi_{n} f_{0}^{(n)}=\delta$ which is new and $w$ is reduced, if case (i) holds then $j=t, \epsilon=\delta$, and $u_{1}=f_{0}^{-1}$, contrary to the assumption that $w$ is cyclically reduced. If case (ii) holds, then as $w^{(n)}$ is defined at $\epsilon$ it follows that each of $u_{j}, \ldots, u_{j+s}$ is equal to $f_{0}$, so $\ell(w) \geq s$. In case (iii) we have $\epsilon^{\prime}=\epsilon=\beta_{k}$, say. Now since $w$ is cyclically reduced and $u_{1}=f_{0}$ and the $\beta_{i}$ are new, $u_{t}=f_{0}$. From this it again follows easily that $\ell(w) \geq s$.

In the second case, we have $u_{j}=f_{0}^{-1}$. Now, one of the following holds.

(i) $\epsilon^{\prime}=\xi_{n}$ and $j=1$ (as $\delta$ is new);

(ii) $\epsilon^{\prime}=\delta$ and $j=1$ (as $\delta$ is new);

(iii) $\epsilon^{\prime} \in\left\{\beta_{1}, \ldots, \beta_{s}\right\}$ and $j=1$.

In case (i), each of $u_{j}, \ldots, u_{j+s}$ equals $f_{0}^{-1}$, so $\ell(w) \geq s$. In case (ii), it follows that $\epsilon^{\prime}=\epsilon=\delta$, and $u_{t}=f_{0}$, contrary to the assumption that $w$ is cyclically reduced. Finally, in case (iii), as the $\beta_{i}$ are new we have $\epsilon^{\prime}=\epsilon=\beta_{k}$, say. Now as in the last paragraph, since $w$ is cyclically reduced it follows that $u_{t}=f_{0}^{-1}$, and $\ell(w) \geq s$.

We have shown that in all cases, if $f_{n}$ does not occur in $w$, then $\ell(w) \geq s \geq n$. Hence, $w$ has just finitely many fixed points, as required.

\section{Proof of Theorem 1.2}

Put $\Omega:=\left\{\xi_{i}: i \in \mathbb{N}\right\}, \Gamma:=\left\{\xi_{2 i}: i \in \mathbb{N}\right\}$, and $\Delta:=\Omega \backslash \Gamma$. Fix a surjection $\Phi: \mathbb{N} \rightarrow \mathbb{N}^{2}$. Let $F_{2}$ be the free group on generators $f, g$. Let $W:=\left\{w_{i}: i \in \mathbb{N}\right\}$ be 
the set of non-empty cyclically reduced words in $f, g, f^{-1}, g^{-1}$. We shall define an action of $F_{2}$ on $\Omega$ step-by-step, so that after step $k$ the partial isomorphisms $f^{(k)}, g^{(k)}$ will have been defined (and $f:=\cup\left(f^{(k)}: k \in \mathbb{N}\right), g:=\cup\left(g^{(k)}: k \in \mathbb{N}\right)$ ). We adopt other notational conventions of Section 1. For each $k \in \mathbb{N}$ there is an equivalence relation $\sim_{k}$ on $\Omega: \alpha \sim_{k} \beta$ if there is some word $w$ such that $\alpha w^{(k)}=\beta$. The $\sim_{k}$-classes will be called $k$-components.

At step 0 , we put $f^{(0)}:=\left\{\left\langle\xi_{0}, \xi_{1}\right\rangle,\left\langle\xi_{1}, \xi_{0}\right\rangle\right\}$ and $g^{(0)}:=\left\{\left\langle\xi_{0}, \xi_{2}\right\rangle,\left\langle\xi_{2}, \xi_{0}\right\rangle\right\}$. We shall preserve throughout the construction the following conditions.

(i) For each $i \in \mathbb{N}$, all partial cycles of $w_{i}^{(k)}$ are finite.

(ii) $\Gamma$ and $\Delta$ are $g^{(k)}$-invariant;

(iii) All $f^{(k)}$-partial cycles other than $\left(\xi_{0}, \xi_{1}\right)$ lie within $\Gamma$ or within $\Delta$.

(iv) If $\Theta$ is a non-empty $k$-component, then there is $\xi \in \Theta$ such that not all of $\xi f^{(k)}, \xi g^{(k)}, \xi\left(f^{(k)}\right)^{-1}, \xi\left(g^{(k)}\right)^{-1}$ are defined (and if $\xi_{0} \in \Theta$ then $\xi$ can be chosen in either $\Gamma$ or $\Delta$ ).

Clearly, the above hold after Step 0 . We also ensure that for each $i>0, w_{i}$ moves some element of $\Omega$, and that $\langle f, g\rangle$ acts transitively on $\Omega$. By (i), part (a) of the theorem holds. By (ii) and (iii), if $x \in F_{2}$ then all but finitely many of the cycles of $x$ lie entirely in $\Gamma$ or entirely in $\Delta$, and (b) of the theorem follows.

The construction is in the following steps.

Step $k=4 n$. Ensure that $\xi_{n} \in \operatorname{dom}\left(f^{(k)}\right) \cap \operatorname{ran}\left(f^{(k)}\right) \cap \operatorname{dom}\left(g^{(k)}\right) \cap \operatorname{ran}\left(g^{(k)}\right)$.

Step $k=4 n+1$. Ensure that $w_{n}^{(k)}$ moves some element of $\Omega$ (to guarantee that $\langle f, g\rangle$ acts faithfully).

Step $k=4 n+2$. Arrange that $\xi_{0}$ and $\xi_{n}$ lie in the same $k$-component (this will yield transitivity of $(f, g)$ on $\Omega$ ).

Step $k=4 n+3$. Ensure that if $\Phi(n)=\langle r, s\rangle$ then the $w_{r}^{(k)}$-cycle containing $\xi_{s}$ is complete (this yields (i) above-the finiteness of all $w$-cycles).

We now verify that each of these steps can be carried out. It is easily checked that (i)-(iv) are preserved.

Step $k=4 n$. Suppose that $\xi_{n} \notin \operatorname{dom}\left(f^{(k-1)}\right) \cup \operatorname{ran}\left(f^{(k-1)}\right)$. Find distinct new points $\xi, \xi^{\prime}$ in $\Gamma$ (if $n$ is even) or in $\Delta$ (if $n$ is odd) and put

$$
f^{(k)}:=f^{(k-1)} \cup\left\{\left\langle\xi, \xi_{n}\right\rangle,\left\langle\xi_{n}, \xi^{\prime}\right\rangle\right\}
$$

There are other cases (when $f$ is replaced by $g$, or when $\xi_{n}$ lies in just one of the domain or range of $f$ or $g$ ), and these are handled similarly.

Step $k=4 n+1$. Let $l:=\ell\left(w_{n}\right)$. We extend $f^{(k-1)}, g^{(k-1)}$ to $f^{(k)}, g^{(k)}$ so that there is an $w_{n}^{(k)}$-chain consisting of distinct new points $\alpha_{0}, \ldots, \alpha_{l} \in \Gamma$ such that $\alpha_{0} w_{n}^{(k)}=\alpha_{l}$.

Step $k=4 n+2$. We may suppose $\xi_{n} \in \Gamma$ (as the case $\xi_{n} \in \Delta$ is essentially the same). Also, we may suppose that $\xi_{0}$ and $\xi_{n}$ are in distinct $(k-1)$-components, as otherwise the result already holds. By (iv), there is $\gamma \in \Gamma$ lying in the $(k-1)$-component 
of $\xi_{0}$ such that for some $h_{1} \in\left\{f, g, f^{-1}, g^{-1}\right\}, \gamma h_{1}^{(k-1)}$ is undefined. Likewise, there is $\delta$ in the $(k-1)$-component of $\xi_{n}$ such that for some $h_{2} \in\left\{f, g, f^{-1}, g^{-1}\right\}, \delta h_{2}^{(k-1)}$ is undefined. Let $h_{3} \in\left\{f, g, f^{-1}, g^{-1}\right\}, h_{3} \neq h_{1}^{-1}, h_{2}$. Choose new points $\epsilon_{1}, \epsilon_{2} \in \Gamma$, and take the least extension of $f^{(k-1)}, g^{(k-1)}$ to $f^{(k)}, g^{(k)}$ so that $\gamma h_{1}^{(k)}=\epsilon_{1}, \epsilon_{1} h_{3}^{(k)}=\epsilon_{2}$ and $\epsilon_{2}\left(h_{2}^{(k)}\right)^{-1}=\delta$. If $w:=h_{1} h_{3} h_{2}^{-1}$, then $\gamma w^{(k)}=\delta$, so $\xi_{0}, \xi_{n}$ are in the same $k$-component, as required.

Step $k=4 n+3$. For notational convenience, put $\xi:=\xi_{s}, w:=w_{r}$ and $l:=\ell(w)$. We may suppose that the $w^{(k-1)}$-cycle containing $\xi$ is incomplete, and has the form $\left(\ldots, \delta_{1}, \ldots, \delta_{1}, \ldots\right)$. (We do not exclude here the case when $\xi$ is a new point, so $t=1$.) Let $u_{1}$ be a maximal initial segment of $w$ such that $\delta_{t} u_{1}^{(k-1)}$ is defined, and likewise let $v_{1}$ be a maximal final segment of $w$ such that $\delta_{1}\left(v_{1}^{(k-1)}\right)^{-1}$ is defined. Put $\epsilon:=\delta_{t} u_{1}^{(k-1)}$ and $\epsilon^{\prime}:=\delta_{1}\left(v_{1}^{(k-1)}\right)^{-1}$. There are words $u_{1}^{\prime}, v_{1}^{\prime}$ so that $w=u_{1} u_{1}^{\prime}=v_{1}^{\prime} v_{1}$ (so $u_{1}^{\prime}, v_{1}^{\prime}$ are non-empty, but possibly equal $w$ ).

Case 1. $\epsilon, \epsilon^{\prime} \in \Gamma$. (The case $\epsilon, \epsilon^{\prime} \in \Delta$ is similar.)

Let $m:=\ell\left(v_{1}^{\prime}\right)+\ell\left(u_{1}^{\prime}\right)$ and put $w^{\prime}:=u_{1}^{\prime} v_{1}^{\prime}$. Then $w^{\prime}$ is reduced, as $w$ is cyclically reduced. Suppose first $\epsilon \neq \epsilon^{\prime}$. Choose new points $\epsilon_{1}, \ldots, \epsilon_{m-1} \in \Gamma$ and extend $f^{(k-1)}, g^{(k-1)}$ so that there is a $w^{\prime(k)}$-chain from $\epsilon$ to $\epsilon^{\prime}$ of the form $\left(\epsilon_{0}, \ldots, \epsilon_{m}\right)$, where $\epsilon_{0}:=\epsilon$ and $\epsilon_{m}:=\epsilon^{\prime}$. The $w$-chain containing $\xi$ is now complete.

If $\epsilon=\epsilon^{\prime}$, slight extra care is needed if some initial segment of $u_{1}^{\prime}$ is equal to an initial segment of $v_{1}^{\prime-1}$. However, as $w$ is cyclically reduced, we cannot have $u_{1}^{\prime}=v_{1}^{\prime-1}$, and so essentially the same argument as above works.

Case 2. $\epsilon^{\prime} \in \Gamma$ and $\epsilon \in \Delta$. (The case $\epsilon^{\prime} \in \Delta$ and $\epsilon \in \Gamma$ is similar.)

In this case, by (ii), there is at least one occurrence of $f$ or $f^{-1}$ in $w$.

CLAIM. There are $\eta \in \Delta$ and $\eta^{\prime} \in \Gamma$ and a maximal $w^{(k-1)}$-chain beginning at $\eta$ and ending at $\eta^{\prime}$ with $\eta\left(u_{2} w^{j} v_{2}\right)^{(k-1)}=\eta^{\prime}$, where $u_{2}$ is a proper final segment of $w$ and $v_{2}$ is a proper initial segment of $w$ (and possibly $j=0$ ).

PROOF OF CLAIM. For each occurrence of $f$ or $f^{-1}$ in $w$, consider the maximal $w^{(k-1)}$-chain in which that occurrence takes $\xi_{0}$ to $\xi_{1}$, and the maximal $w^{(k-1)}$-chain in which that occurrence takes $\xi_{1}$ to $\xi_{0}$. Let $C_{1}, \ldots, C_{t}$ list the $w^{(k-1)}$-chains so obtained. Let $a_{i}$ be the number of $(\Gamma, \Delta)$-crossings of $C_{i}$ (that is, successive pairs $\xi_{0}, \xi_{1}$ in $C_{i}$ ), and $b_{i}$ the number of $(\Delta, \Gamma)$-crossings (successive pairs $\left.\xi_{1}, \xi_{0}\right)$. Each occurrence of $f$ (or $f^{-1}$ ) in $w$ determines a unique $(\Gamma, \Delta)$-crossing of some $C_{i}$, and a unique $(\Delta, \Gamma$ ) of some (distinct) $C_{j}$. Also each (oriented) crossing of each $C_{i}$ comes from a unique occurrence of $f$ or $f^{-1}$ in $w$. It follows that

$$
a_{1}+\cdots+a_{t}=b_{1}+\cdots+b_{t} .
$$

If $C_{i}$ is a chain of a complete $w^{(k-1)}$-cycle, or begins and ends in $\Gamma$, or begins and ends in $\Delta$, then $a_{i}=b_{i}$. Likewise, if $C_{i}$ begins in $\Gamma$ and ends in $\Delta$ then $a_{i}=b_{i}+1$, and if 
$C_{i}$ begins in $\Delta$ and ends in $\Gamma$ then $b_{i}=a_{i}+1$. Since the chain from $\epsilon^{\prime}$ to $\epsilon$ begins in $\Gamma$ and ends in $\Delta$, it follows that there is some $C_{i}$ which begins in $\Delta$ and ends in $\Gamma$, as required.

Given the claim, write $w=u_{2}^{\prime} u_{2}=v_{2} v_{2}^{\prime}$. As in case 1, the words $u_{1}^{\prime} u_{2}^{\prime}$ and $v_{2}^{\prime} v_{1}^{\prime}$ are reduced. Suppose first that $\epsilon \neq \eta$ and $\epsilon^{\prime} \neq \eta^{\prime}$. As in Case 1 , extend $f^{(k-1)}, g^{(k-1)}$ to $f^{(k)}, g^{(k)}$ in a minimal way, entirely using new points, so that $\epsilon\left(u_{1}^{\prime} u_{2}^{\prime}\right)^{(k)}=\eta$ and $\eta^{\prime}\left(v_{2}^{\prime} v_{1}^{\prime}\right)^{(k)}=\epsilon^{\prime}$. As in Case 1, slight extra care is needed if say $\epsilon=\eta$ (and similarly if $\epsilon^{\prime}=\eta^{\prime}$ ). For example, it could happen that $\epsilon=\eta$ and there is an initial segment $u$ of $u_{1}^{\prime}$ such that $u^{-1}$ is a final segment of $u_{2}^{\prime}$. In this case, since $w$ is cyclically reduced we cannot have $u_{1}^{\prime}=u=\left(u_{2}^{\prime}\right)^{-1}$, and it follows that the extension is still possible.

REMARK 3.1. There are certain refinements of the construction in the proof of Theorem 1.2. For example, it is possible to arrange that $F_{2}$ acts 2-transitively on $\Omega$. One needs to show that the stabiliser of $\xi_{2}$ can be made transitive on $\Omega \backslash\left\{\xi_{2}\right\}$. The idea is, for an arbitrary $\xi$, to fix $\xi_{2}$ and map $\xi_{0}$ to $\xi_{i}$ by some very long word. More generally, one can arrange that the action of $F_{2}$ on $\Omega$ is highly transitive, that is, $k$-transitive for all $k>0$.

\section{Proof of Theorem 1.3}

We build automorphisms $f, g$ of the random graph $(\Omega, \sim)$. Let $S$ be the set of non-empty cyclically reduced words in $f, g, f^{-1}, g^{-1}$. Define an equivalence relation $\equiv$ on $S$, putting $\boldsymbol{u} \equiv v$ if and only if there are words $w_{1}, w_{2} \in S$ and $r, s \in \mathbb{Z} \backslash\{0\}$ such that

$$
w_{1}^{-1} u^{r} w_{1}=w_{2}^{-1} v^{s} w_{2}
$$

Let $W=\left\{w_{i}: i \in \mathbb{N}\right\}$ consist of exactly one element, chosen of least possible length, from each $\equiv$-class. By the minimality assumption no element of $W$ can be a proper power, and each is reduced, and not conjugate to any shorter word. The construction of $f, g$ is by finite approximation, and after step $k$ we denote by $f^{(k)}, g^{(k)}$ the restrictions of $f, g$ so far defined (likewise, for any word $w, w^{(k)}$ is the restriction defined after step $k$ ). Let $l_{n}:=\ell\left(w_{n}\right)$. We suppose that $w_{0}=f$ and $w_{1}=g$. To ensure that $\langle f, g\rangle$ generate a ZTF group, we shall arrange that each $w_{i}$ has just finitely many cycles. One of the steps will be to extend $f^{(k)}, g^{(k)}$ so that certain partial cycles of some $w_{i}$ are 'joined' into a single cycle. This is not always possible: for example, if a partial automorphism $h$ had incomplete cycles $\left(\ldots, \alpha_{1}, \ldots, \alpha_{r}, \ldots\right)$ and $\left(\ldots, \beta_{1}, \ldots, \beta_{s}, \ldots\right)$ where $\alpha_{1} \sim \alpha_{2}$ but $\beta_{1} \not \beta_{2}$, then there is no extension of $h$ with a single cycle extending these partial cycles. This problem did not arise in [7] 
(where there was no invariant relational structure) and makes the proof here more complicated. First, we must formalise the notion of compatibility of partial cycles.

DEFINITION 4.1. Let $w$ be a word, with $\ell(w)=l$. Then two $w^{(k)}$-cycles

$$
\left(\ldots, \epsilon_{1}, \ldots, \epsilon_{r}, \ldots\right) \text { and }\left(\ldots, \epsilon_{1}^{\prime}, \ldots, \epsilon_{s}^{\prime}, \ldots\right)
$$

are compatible, if the following hold, where the $w^{(k)}$-chains corresponding to the above cycles are $\left(\delta_{1}, \ldots, \delta_{n}\right)$ and $\left(\delta_{1}^{\prime}, \ldots, \delta_{m}^{\prime}\right)$.

(i) For all $t \in \mathbb{N}$ with $t l \leq \min \{m, n\}$

$$
\delta_{1} \sim \delta_{t l} \leftrightarrow \delta_{1}^{\prime} \sim \delta_{t l}^{\prime} .
$$

(ii) Suppose the finite complete $w^{(k)}$-cycles are $C_{1}, \ldots, C_{p}$, of lengths $r_{1}, \ldots, r_{p}$, and that $D_{i}:=\left\{x \in C_{i}: x \sim \epsilon_{1}\right\}$ and $D_{i}^{\prime}:=\left\{x \in C_{i}: x \sim \epsilon_{1}^{\prime}\right\}$. Then there are $s_{1}, \ldots, s_{p} \in \mathbb{N}$ such that $D_{i}^{\prime}=D_{i}\left(w^{(k)}\right)^{s_{i}}$ for each $i$ and there is $a \in \mathbb{N}$ such that $a \equiv s_{i}\left(\bmod r_{i}\right)$ for each $i=1, \ldots, p$.

We shall say that two $w^{(k)}$-cycles are weakly compatible if just condition (ii) above holds.

Because of condition (i), compatibility is not an equivalence relation (since two incompatible long $w^{(k)}$-cycles can each be compatible with a short $w^{(k)}$-cycle). However, weak compatibility is an equivalence relation. The idea of the above definition is that if two $w^{(k)}$-cycles are compatible then it should be possible to extend $w^{(k)}$ so that they are parts of a single cycle and such that the number of new points used to join the two cycles depends on the $a$ obtained in (ii) above. We shall do this explicitly a little later.

We now describe the construction of $f, g$. First, we fix a surjective function $\Phi: \mathbb{N} \rightarrow \mathbb{N}^{3}$ which takes each value of $\mathbb{N}^{3}$ infinitely often. Our construction proceeds through steps $5 n$ to $5 n+4$. If $k \in\{5 n, \ldots, 5 n+4\}$, then step $k$ may be a sequence of substeps. We adopt the general notation that for a word $w$, the function determined by $w$ before such a substep of step $k$ is written $w^{(k *)}$, and after the substep it is denoted by $w^{(k+)}$.

Definition 4.2. Suppose $a, b \in \mathbb{N}$ with $a, b<k / 5$, and that $w_{a}, w_{b} \in W$. A $\left(w_{a}, w_{b}, k *, \alpha, \beta\right)$-coincidence consists of a $w_{a}^{(k *)}$-chain and a distinct $w_{b}^{(k *)}$-chain from $\alpha$ to $\beta$ such that there is a common letter $x$ (one of $f, g, f^{-1}$, or $g^{-1}$ ) such that $\beta x^{(k *)}$ is undefined, but in some extension of both the $w_{a}^{(k *)}$-chain and the $w_{b}^{(k *)}$-chain, $\beta x^{(k+)}$ would be the next element after $\beta$.

Before starting step $5 n$, we partition the incomplete cycles of $w_{n-1}^{(5 n-1)}$ which lie in maximal chains of length at least $2 l_{n-1}$ into finitely many classes, say

$$
K_{1}^{n-1}, \ldots, K_{h(n-1)}^{n-1},
$$


so that any two cycles in some $K_{i}^{n-1}$ are compatible (here $h: \mathbf{N} \rightarrow \mathbf{N}$ is some indexing function). We will eventually arrange that $w_{n-1}$ has $h(n-1)$ infinite cycles (so that $h(0)=1$ and $h(1)=2$ ), with partial cycles in a given $K_{i}^{n-1}$ eventually being joined so they lie in the same cycle. The word $w_{n-1}$ acquires no new finite complete cycles after step $5 n-1$. If a partial cycle lies in $K_{i}^{n-1}$ then we refer to $K_{i}^{n-1}$ as its type, or compatibility type. This is never changed: later extensions of a partial cycle in $K_{i}^{n-1}$ will still have type $K_{i}^{n-1}$, and at later stages, every cycle of $w_{n-1}^{(k)}$ in a maximal chain of length at least $2 l_{n-1}$ will have type $K_{i}^{n-1}$ for some unique $i \in\{1, \ldots, h(n-1)\}$. At each substep after $5 n$, as soon as a partial cycle of $w_{n-1}$ lies in a chain of length at least $2 l_{n-1}$, we choose some $i$ so that the cycle is compatible with cycles of type $K_{i}^{n-1}$, and specify that it has type $K_{i}^{n-1}$. At any stage any two cycles of a word of the same type will be compatible.

Step $k=5 n$. Ensure that $\xi_{n} \in \operatorname{dom}\left(f^{(k)}\right) \cap \operatorname{ran}\left(f^{(k)}\right)$.

Step $k=5 n+1$. Ensure that $\xi_{n} \in \operatorname{dom}\left(g^{(k)}\right) \cap \operatorname{ran}\left(g^{(k)}\right)$.

Step $k=5 n+2$. If $\Phi(n)=(p, q, r)$ with $p<n$, and $\xi_{q}, \xi_{r}$ lie in $w_{p}^{(k-1)}$-cycles of the same type, each in chains of length at least $2 l_{p}$, extend $f^{(k-1)}, g^{(k-1)}$ so that $\xi_{q}, \xi_{r}$ are in the same $w_{p}^{(k)}$-cycle.

Step $k=5 n+3$. We ensure that over the complete cycles of $w_{n}^{(k-1)}$ there are $w_{n}^{(k)}$-cycles of each possible weak compatibility class lying in chains of length at least $2 l_{n}$.

Step $k=5 n+4$. Extend $f^{(k-1)}, g^{(k-1)}$ to arrange that there are no $\left(w_{i}, w_{n}, 5 n+4\right.$, $\alpha, \beta)$-coincidences for $i \leq n$.

Throughout the steps $5 n$ to $5 n+4$, we ensure that

(a) up to compatibility there is a unique $f^{(k)}$-cycle and at most 2 incomplete $g^{(k)}$ cycles, and $g^{(k)}$ has a unique complete cycle $\left(\xi_{0}\right)$.

(b) there is no coincidence in which both the words involved are from $w_{0}, \ldots, w_{n-1}$,

(c) if $i \leq n-1$, then any complete $w_{i}^{(5 n+4)}$-cycle is a complete $w_{i}^{(5 n-1)}$-cycle,

(d) any extension of cycles of $w_{i}^{k *}(0 \leq i \leq n-1)$ respects their compatibility type; that is if two cycles of $w_{i}^{(k *)}$ have the same type, then so do their extensions to cycles of $w_{i}^{(k+)}$.

We call any extension $f^{(k+)}, g^{(k+)}$ of $f^{(k *)}, g^{(k *)}$ preserving these properties a good extension.

LEMMA 4.3. Suppose that $n=[k / 5]$, the integer part of $k / 5$, and that $\gamma \notin$ $\operatorname{dom}\left(f^{(k *)}\right)$. Suppose that after step $k *$, (a)-(d) above hold. Then there is $\delta \in \Omega$ so that the extension $f^{(k+)}:=f^{(k *)} \cup\{\langle\gamma, \delta\rangle\}, g^{(k+)}:=g^{(k *)}$ is good.

REMARK. The corresponding statements hold with $f^{-1}, g$, or $g^{-1}$ in place of $f$.

Proof of LemMa 4.3. We must choose $\delta$, a new point of $\Omega$. The requirement 
that $f^{(k+)}$ is a partial automorphism essentially says that for certain finite disjoint $\Delta_{1}, \Delta_{2} \subseteq \Omega, \delta$ must be chosen in

$$
\left\{x: \forall y \in \Delta_{1}(x \sim y) \wedge \forall y \in \Delta_{2}(x \not y)\right\} .
$$

By the extension property which characterises the random graph, this set is infinite, that is, there are infinitely many choices for such $\delta$. As $\delta$ is new and the $w_{j}$ are cyclically reduced, for any $i \in \mathbb{N}$ any complete cycle of $w_{i}^{(k+)}$ is a complete cycle of $w_{i}^{(k *)}$, so in particular (c) above will be satisfied.

We next check that $\delta$ can be found so that (d) holds. Condition (ii) in Definition 4.1 plays no role here, because the weak compatibility class of a partial cycle is determined by one of its elements. Essentially, our compatibility requirements merely force us to restrict the choice of $\delta$ by increasing $\Delta_{1}$ and $\Delta_{2}$ (to ensure that the conditions are satisfied). The only problem is to ensure that $\Delta_{1}$ and $\Delta_{2}$ are disjoint, that is, that it doesn't happen that one compatibility requirement puts some $\epsilon \epsilon \Delta_{1}$, and some other compatibility (or automorphism) condition puts $\epsilon \in \Delta_{2}$. There could not be a clash between a compatibility requirement and an automorphism condition, for suppose the compatibility requirement forced $\delta \sim \epsilon$ (that is, $\epsilon \in \Delta_{1}$ ) and an automorphism requirement forced $\delta \not \epsilon$ (that is, $\epsilon \in \Delta_{2}$ ). This means that for some $a \leq n$ there will be a $w_{a}^{(k+)}$-chain of length $t l_{a}$ say from $\epsilon$ to $\delta$, and further $\eta:=\epsilon\left(f^{(k *)}\right)^{-1} \chi \gamma$. However, in this case there is already a $w_{a}^{(k *)}$-chain of length $t l_{a}$ from $\eta$ to $\gamma$ which conflicts with our compatibility requirements. It can be checked that two compatibility requirements can only clash if there was a $\left(w_{a}, w_{b}, k *, \alpha, \beta\right)$-coincidence, and by assumption there is none.

To verify (b), suppose that there is a $\left(w_{a}, w_{b}, k+, \alpha, \beta\right)$ coincidence. Then either $\alpha=\delta$ or $\beta=\delta$. If $\beta=\delta$, then the last letter used in both the $w_{a}^{(k+)}$-chain and the $w_{b}^{(k+)}$-chain is $f$, so there was previously a $\left(w_{a}, w_{b}, k *, \alpha, \gamma\right)$-coincidence, contrary to (b) at the previous step. Similarly, if $\delta=\alpha$, then there was previously a $\left(w_{a}, w_{b}, k *, \gamma, \beta\right)$-coincidence, again a contradiction.

LEMMA 4.4. (i) Let $w_{a}, w_{b} \in W$, with $w_{a} \not \equiv w_{b}$, and put $l_{a}=\ell\left(w_{a}\right)$, $l_{b}=\ell\left(w_{b}\right)$. Suppose that $w_{a}, w_{b}$ have a common chain of length $n$. Then

$$
n<\max \left\{l_{a}\left(l_{a}+1\right), l_{b}\left(l_{b}+1\right)\right\}
$$

(ii) Let $w \in W$ have length $l$ and $\left(\delta_{0}, \ldots, \delta_{2 l}\right)$ be a $w$-chain with $\delta_{0} w^{2}=\delta_{2 l}$. Suppose that for some $i>0$ there is a w-chain $\left(\delta_{i}, \ldots, \delta_{i+l}\right)$. Then $i=l$.

Proof. (i) Suppose not. We may suppose $l_{a} \geq l_{b}$. By the pigeon-hole principle, we may suppose there are distinct $\epsilon_{i}, \epsilon_{j}$ on the $w_{a}$-cycle and an initial subword $u$ of $w_{a}$ such that $\epsilon_{i} u, \epsilon_{j} \boldsymbol{u}$ are on the $w_{b}$-cycle. (There is another possible case, handled 
similarly, when $\boldsymbol{u}$ is a final subword of $w_{a}$ and $\epsilon_{i} u^{-1}, \epsilon_{j} u^{-1}$ are on the $w_{b}$-cycle.) Hence $\epsilon_{i} u w_{b}^{k}=\epsilon_{j} u$ for some $k \in \mathbb{N}$. Put $m:=j-i$. Then $\epsilon_{i} w_{a}^{m}=\epsilon_{j}$ (via the same chain), so $w_{a}^{m} u=u w_{b}^{k}$. Hence $w_{a} \equiv w_{b}$, which is a contradiction.

(ii) Suppose $i \neq l$ and suppose $u, v$ are respectively initial and final segments of $w$ such that $\delta_{0} u=\delta_{i}$ and $\delta_{i+l} v=\delta_{2 l}$. Then (by considering lengths of words), $\delta_{i} v=\delta_{l}$ and $\delta_{l} u=\delta_{i+l}$. It follows that $u w=w u$, so $w$ is a proper power of $u$, which is impossible.

To get started we write down the first 10 steps explicitly (remembering that $w_{0}=f$, $w_{1}=g$ ). This will serve to check that the conditions hold early on.

Step 0: Put $f^{(0)}=\left(\ldots \xi_{1}, \xi_{0}, \xi_{i} \ldots\right)$, where $i \in \mathbb{N} \backslash\{0,1\}$ is least such that $\xi_{1} \sim \xi_{0} \leftrightarrow \xi_{0} \sim \xi_{i}$. As in Section 2 this notation means that $\left(\ldots \xi_{1}, \xi_{0}, \xi_{i} \ldots\right)$ is a partial cycle of $f^{(0)}$, so $\xi_{1} f^{(0)}=\xi_{0}$ and $\xi_{0} f^{(0)}=\xi_{i}$, with $f^{(0)}$ not defined elsewhere.

Step 1: Put $g^{(0)}=\left(\xi_{0}\right)$. That is $g$ fixes $\xi_{0}$.

It is easy to see that there is nothing to be done in Steps 2, 3 and 4. At this stage we specify that $h(0)=1$, that is, $w_{0}=f$ has a unique compatibility type $K_{1}^{0}$.

Step 5: Put $f^{(5)}=\left(\ldots \xi_{j}, \xi_{1}, \xi_{0}, \xi_{i} \ldots\right)$, where $j \in \mathbb{N} \backslash\{0,1, i\}$ is least such that $\xi_{j} \sim \xi_{1} \leftrightarrow \xi_{1} \sim \xi_{0}$ and $\xi_{j} \sim \xi_{0} \leftrightarrow \xi_{1} \sim \xi_{i}$.

Step 6: Here we need to put $\xi_{1}$ into the domain and range of $g$. Let us call a point $\xi$ of $\Omega$ a neighbour of $\xi_{0}$ if $\xi \sim \xi_{0}$ and a non-neighbour otherwise. Since $g$ fixes $\xi_{0}$ we extend $g$ in such a way that all neighbours will eventually be in one cycle and the nonneighbours in another, thus giving us 3 cycles in all. Put $g^{(5)}=\left(\xi_{0}\right)\left(\ldots \xi_{n}, \xi_{1}, \xi_{m} \ldots\right)$ where $\xi_{n}, \xi_{1}, \xi_{m}$ are either all neighbours or all non-neighbours of $\xi_{0}$ and

$$
\xi_{n} \sim \xi_{1} \leftrightarrow \xi_{1} \sim \xi_{m} .
$$

Step 7: Nothing need be done, as $g^{(6)}$ has a unique incomplete cycle with more than one point.

Step 8: The only complete cycle of $g^{(7)}$ is $\left(\xi_{0}\right)$, so for example if $\xi_{1} \sim \xi_{0}$, then at step 8 we must extend $g^{(7)}$ by adjoining an incomplete 2-cycle of non-neighbours of $\xi_{0}$.

Step 9: Nothing need be done, as $w_{0}, w_{1}$ have length 1 and distinct words of length 1 cannot have a common next letter as required for a coincidence.

Finally, we specify that $w_{1}=g$ has two compatibility types $K_{1}^{1}$ and $K_{2}^{1}$, corresponding to neighbours and non-neighbours of $\xi_{0}$ respectively, so $h(1)=2$.

It follows immediately from Lemma 4.3 and the remark following its statement that Steps $5 n$ and $5 n+1$ are possible. It is also straightforward to see that Step $5 n+3$ is possible, since we can construct new $w_{n}^{(5 n+3)}$-chains of length $l_{n}$ using new points.

Step $k=5 n+2$. This is the most troublesome step. Suppose that $\Phi(n)=$ $\left(q_{1}, q_{2}, q_{3}\right)$ with $q_{1}<n$ and write $w:=w_{q_{1}}$ and $l:=\ell(w)$. Let $\left(\ldots, \epsilon_{1}, \ldots, \epsilon_{c}, \ldots\right)$ be the $w^{(k-1)}$-cycle containing $\xi_{q_{2}}$, with corresponding $w^{(k-1)}$-chain $\left(\delta_{1}, \ldots, \delta_{r}\right)$, and 
$\left(\ldots, \epsilon_{1}^{\prime}, \ldots, \epsilon_{d}^{\prime}, \ldots\right)$ be the $w^{(k-1)}$-cycle containing $\xi_{q_{2}}$, with corresponding $w^{(k-1)}$ chain $\left(\delta_{1}^{\prime}, \ldots, \delta_{s}^{\prime}\right)$. We may suppose that these two cycles are distinct, as otherwise there is nothing to do. By extending these two cycles if necessary (using Lemma 4.3), we may assume that they are the two longest $w^{(k-1)}$-cycles, with $c>d$.

Let $l^{\prime}:=\max \left\{l_{0}, \ldots, l_{n}\right\}$. We first apply Lemma 4.3 to add between $l^{2}+l^{\prime}$ and $l^{\prime 2}+2 l^{\prime}$ new points to each end of the $w^{(k-1)}$-chain $\left(\delta_{1}, \ldots, \delta_{r}\right)$ to obtain a new $w^{(k *)}$ chain $\left(\alpha_{1}, \ldots, \alpha_{r^{\prime}}\right)$ with $w$-cycle $\left(\ldots, \epsilon_{-m}, \ldots, \epsilon_{c+m+1}, \ldots\right)$, where $\alpha_{1}=\epsilon_{-m}$ and $\alpha_{r^{\prime}}=\epsilon_{c+m+1}\left(\right.$ so $\left.m \geq l^{\prime}\right)$. Likewise, we can find a good extension of the $w^{(k-1)}$-chain $\left(\delta_{1}^{\prime}, \ldots, \delta_{s}^{\prime}\right)$ to a chain $\left(\alpha_{1}^{\prime}, \ldots, \alpha_{s^{\prime}}^{\prime}\right)$ with $w^{(k *)}$-cycle $\left(\ldots, \epsilon_{-m}^{\prime}, \ldots, \epsilon_{d+m+1}^{\prime}, \ldots\right)$, where $\alpha_{1}^{\prime}=\epsilon_{-m}^{\prime}$ and $\alpha_{s^{\prime}}^{\prime}=\epsilon_{d+m+1}^{\prime}$. This is done so Lemma 4.4 (i) can be applied later.

We now adopt the notation of Definition 4.1 for the complete cycles $C_{1}, \ldots, C_{p}$ of $w^{(k-1)}$. In particular, $D_{i}:=\left\{x \in C_{i}: x \sim \epsilon_{1}\right\}$ and $D_{i}^{\prime}=\left\{x \in C_{i}: x \sim \epsilon_{1}^{\prime}\right\}$, for each $i=1, \ldots, p$. By compatibility, there is $a$ such that $a \equiv s_{i}\left(\bmod r_{i}\right)$ for each $i=1, \ldots, p$. Put $b:=a-(c+2 m+1)$. The idea here is to ensure that $\epsilon_{1} w^{a}=\epsilon_{1}^{\prime}$. Now $\epsilon_{1} w^{c+m}=\epsilon_{c+m+1}$, and $\epsilon_{-m}^{\prime} w^{m+1}=\epsilon_{1}^{\prime}$. Thus we need $b$ such that $\epsilon_{c+m+1} w^{b}=\epsilon_{-m}^{\prime}$. That gives $a=c+m+b+m+1$. For later convenience, we choose $a$ so that $b>c+2 m+2$.

We shall find new $\gamma_{1}, \ldots, \gamma_{l b-1}$ so that there is a good extension $f^{(k+)}, g^{(k+)}$ of $f^{(k *)}, g^{(k *)}$ such that there is a $w^{(k+)}$-chain $\left(\alpha_{1}, \ldots, \alpha_{r^{\prime}}, \gamma_{1}, \ldots, \gamma_{l b-1}, \alpha_{1}^{\prime}, \ldots, \alpha_{s^{\prime}}^{\prime}\right)$ with $\alpha_{r^{\prime}}\left(w^{(k+)}\right)^{b}=\alpha_{1}^{\prime}$. To smooth out notation, we put

$$
\gamma_{-\left(r^{\prime}-1\right)}:=\alpha_{1}, \ldots, \gamma_{0}:=\alpha_{r^{\prime}}, \gamma_{l b}:=\alpha_{1}^{\prime}, \ldots, \gamma_{l b+s^{\prime}-1}:=\alpha_{s^{\prime}}^{\prime}
$$

The process is inductive. After a typical step $k *$ we will have found $\gamma_{1}, \ldots, \gamma_{i-1}$, so that $\left(\gamma_{-\left(r^{r}-1\right)}, \ldots, \gamma_{i-1}\right)$ is a $w^{(k *)}$-chain. At step $k+$ we must find $\gamma_{i}$ so that the following conditions hold (they are assumed inductively to hold after step $k *$ ). Below, we say that a word $z$ potentially takes $\alpha$ to $\beta$ if, for any extension of $f^{(k+)}, g^{(k+)}$ to $f^{(k)}, g^{(k)}$ (partial permutations, not necessarily automorphisms) given by choosing $\gamma_{i+1}, \ldots, \gamma_{l b-1}$ so that $\left(\gamma_{-\left(r^{\prime}-1\right)}, \ldots, \gamma_{l b+s^{\prime}-1}\right)$ is a $w^{(k)}$-chain, we have $\alpha z^{(k)}=\beta$. Thus, for example, before finding $\gamma_{1}$ the word $w^{b}$ potentially takes $\gamma_{0}$ to $\gamma_{l b}$, and if $f$ is the first letter of $w$, then $w^{b} f$ potentially takes $\gamma_{0}$ to $\gamma_{l b+1}$. The idea of (1)-(4) below is that we have an implicit commitment that a certain final subword of $w^{b}$ must eventually take $\gamma_{i}$ to $\gamma_{i b}$. We will also sometimes say that a word $z$ will eventually take $\alpha$ to $\gamma_{j} \in\left\{\gamma_{i+1}, \ldots, \gamma_{l b-1}\right\}$, or write that $\alpha z^{(k)}=\gamma_{j}$, meaning that for any extension $f^{(k)}, g^{(k)}$ as above, we have $\alpha z^{(k)}=\gamma_{j}$.

(1) Automorphism conditions: $f^{(k+)}, g^{(k+)}$ are partial automorphisms.

(2) Compatibility conditions: for $i<n$, if two partial cycles of $w_{i}^{(k *)}$ have the same compatibility type, so do their extensions after step $k+$.

(3) If $\alpha, \beta \in \Omega$ with $\alpha z^{(k+)}=\beta$, and $\mu, \lambda \in \Omega$ and $\mu z^{(k+)}$ is undefined but $z$ potentially takes $\mu$ to $\lambda, \mu \sim \alpha \leftrightarrow \lambda \sim \beta$. 
(4) If $\alpha, \beta, \alpha^{\prime}, \beta^{\prime} \in \Omega$ and $\alpha z^{(k+)}, \beta z^{(k+)}$ are undefined but $z$ potentially takes $\langle\alpha, \beta\rangle$ to $\left\langle\alpha^{\prime}, \beta^{\prime}\right\rangle$, then $\alpha \sim \alpha^{\prime} \leftrightarrow \beta \sim \beta^{\prime}$.

Conditions (3) and (4) deal with commitments arising because of the intention later to add $\gamma_{i+1}, \ldots, \gamma_{l b-1}$. They become important when we choose $\gamma_{l b-1}$. Up until then, using Lemma 4.3, we could make choices preserving just (1) and (2). However, to ensure that we can choose $\gamma_{l b-1}$ so that (1) and (2) still hold, we need to preserve (3) and (4) throughout the construction (and this will suffice). To see this, suppose after step $(k *)$ we have found $\gamma_{1}, \ldots, \gamma_{l b-2}$, and must find $\gamma_{l b-1}$, subject say to $\gamma_{l b-2} f^{(k+)}=\gamma_{l b-1}$ and $\gamma_{l b-1} g^{(k+)}=\gamma_{l b}$. Suppose say that it is impossible to find $\gamma_{l b-1}$ (subject to (1) above). In this case there are $\lambda, \mu, \nu$ such that $\lambda f^{(k *)}=\mu$ and $\lambda \nsim \gamma_{l b-2}$, and $\mu g^{(k *)}=\nu$ and $\nu \sim \gamma_{l b}$ (or the same holds with $\sim$ and $\not$ reversed). Then after step $(k *)$ we had that $f g$ potentially takes $\gamma_{l b-2}$ to $\gamma_{l b}$, and $\lambda f^{(k *)} g^{(k *)}=\nu$, but $\lambda \nsim \gamma_{l b-2}$ but $\nu \sim \gamma_{l b}$, contrary to (3) or (4) at step $k *$.

Condition (2) above poses no problems, essentially by Lemma 4.4 (i) (and the fact that we extended the chain $\left(\delta_{1}, \ldots, \delta_{r}\right)$ sufficiently). For when we add $\gamma_{1}, \ldots, \gamma_{l b-1}$, no cycles for $w_{0}, \ldots, w_{n-1}$, other than the obvious one for $w$, are affected (we may create some new cycles for other words, but they will be compatible with previous cycles). Also, as in the proof of Lemma 4.3, we may at each stage choose $\gamma_{i}$ such that (1) holds (with the argument in the last paragraph for $i=l b-1$ ). Thus, the problem is to show that (3) and (4) hold before $\gamma_{1}$ is chosen, and that, assuming that $\gamma_{1}, \ldots, \gamma_{i-1}$ are chosen to satisfy (1)-(4) and that the choice of $\gamma_{i}$ also satisfies (1), then it can be arranged that (3) and (4) also hold after the choice of $\gamma_{i}$.

We first simplify (3). Suppose that $\boldsymbol{u}^{(k+)}$ is the word which will take $\gamma_{0}$ along the chain $\left(\gamma_{0}, \gamma_{1}, \ldots, \gamma_{i}\right)$ to $\gamma_{i}$, and that $w^{b}=u v$ (so that $v$ potentially takes $\gamma_{i}$ to $\gamma_{l b}$ ). Then, since we assume $\gamma_{i}$ is chosen to satisfy (1), an easy induction argument on the length of $z$ in (3) allows us to assume that $z=v$, with $\alpha=\gamma_{i}$ and $\beta=\gamma_{l b}$. We omit the other case, when $z=v^{-1}$ and $\alpha=\gamma_{i b}, \beta=\gamma_{0}$.

Starting the induction. First, note that condition (3) holds before $\gamma_{1}$ is chosen, since the $w^{(k *)}$-cycles

$$
\left(\ldots, \gamma_{-\left(r^{\prime}-1\right)}, \ldots, \gamma_{0}, \ldots\right) \text { and }\left(\ldots, \gamma_{l b}, \ldots, \gamma_{l b+s^{\prime}-1}, \ldots\right)
$$

satisfy condition (ii) of Definition 4.1 , and since by the choice of $b$, there are no other $w$-cycles of length $b$.

We show now that (4) holds at the beginning, that is, when $(k+)$ is the step before $\gamma_{1}$ is chosen. Suppose not, and let the word $z$ be a counterexample to (4) of least length. Then we can write $z$ as $x_{1} y_{1} x_{2} y_{2} \ldots x_{p} y_{p}$, where the $x_{i}, y_{i}$ are reduced words ( $y_{p}$ possibly empty, the other $y_{i}$ non-empty), with $x_{1}, \ldots, x_{r} \in\left\{w^{b}, w^{-b}\right\}$. Furthermore, we may suppose $\alpha \in\left\{\gamma_{0}, \gamma_{l b}\right\}$, say $\alpha=\gamma_{0}$, in which case $x_{1}=w^{b}$. Slightly abusing notation, we shall write that $\alpha x_{1}^{(k+)}=\gamma_{l b}$. Since we are reducing to 
the case $p=1$, we first suppose $p>1$. Each $y_{i}^{(k+)}$ is defined on $\alpha\left(x_{1} y_{1} \cdots x_{i-1}\right)^{(k+)}$, and $\alpha\left(x_{1} y_{1} \cdots x_{i-1}\right)^{(k+)}$ is equal to $\gamma_{l b}$ if $x_{i-1}=w^{b}$, or $\gamma_{0}$ if $x_{i-1}=w^{-b}$. By minimality of $z$ (using (1) and that (3) holds at the beginning), $\alpha^{\prime} x_{1}^{(k+)}, \alpha^{\prime}\left(x_{1} y_{1}\right)^{(k+)}$ are undefined, that is, $x_{1}, x_{1} y_{1}$ will eventually take $\alpha^{\prime}$ to points in $\left\{\gamma_{1}, \ldots, \gamma_{l b-1}\right\}$. Since $x_{1}$ ends in a copy of $w$, and $x_{2}$ starts with a copy of $w$ or $w^{-1}$, it follows by Lemma 4.4 (ii) that

$$
\alpha^{\prime} x_{1}^{(k)}, \alpha^{\prime}\left(x_{1} y_{1}\right)^{(k)} \in\left\{\gamma_{0} w^{i}: i=1, \ldots, b-1\right\}
$$

In particular, $y_{1}$ is a power of $w$ (clearly a positive power as it is defined on $\left.\gamma_{l b}=\alpha x_{1}^{(k+)}\right)$. However, in this case $\gamma_{l b} y_{1}=\gamma_{l(b+j)}$ for some $j>0$, contradicting that $\gamma_{l b} y_{1} \in\left\{\gamma_{0}, \gamma_{l b}\right\}$

Thus, we have $p=1$, so $z=w^{b} y_{1}$, with $\gamma_{l b} y_{1}$ defined after step $(k+)$. By minimality of $z$, we also have $z=y_{1}^{\prime} w^{b}$ or $z=y_{1}^{\prime} w^{-b}$, and we suppose the former (the latter is similar). Then $\beta^{\prime}=\gamma_{l b}$. We wish to show $y_{1}$ or $y_{1}^{\prime}$ is a power of $w$, for then (4) holds by condition (i) of Definition 4.1.

Suppose first $l\left(y_{1}^{\prime}\right) \geq l b$. Then, since $w^{b}$ is an initial segment of $z$, we have $z=w^{b} u w^{b}$ for some $u$. In this case, as $w^{b}$ is defined (before the choice of $\gamma_{1}$ ) on $\gamma_{l b} u^{(k *)}$, the element $\gamma_{l b} u^{(k *)}$ lies on a finite complete $w$-cycle, as does $\alpha\left(u^{-1}\right)^{(k *)}$. Now $\alpha \sim \alpha^{\prime} \leftrightarrow \beta \sim \alpha^{\prime}$ (as the two $w$-cycles being joined satisfy Definition 4.1 (ii)). Since $u$ is defined on $\alpha^{\prime}, \beta$ before $\gamma_{1}$ is chosen, $\beta \sim \alpha^{\prime} \leftrightarrow \beta^{\prime} \sim \alpha$. By Definition 4.1 (ii) again, $\beta^{\prime} \sim \alpha \leftrightarrow \beta^{\prime} \sim \beta$, whence $\alpha \sim \alpha^{\prime} \leftrightarrow \beta \sim \beta^{\prime}$, as required. Thus, we may assume $\ell\left(y_{1}^{\prime}\right)<l b$, so $y_{1}^{\prime}$ is an initial subword of $w^{b}$ (and likewise $y_{1}$ is a final subword). It now follows by Lemma 4.4 (ii) that $y_{1}^{\prime}$ is a power of $w$, since otherwise $z$ would not potentially take $\alpha$ to $\beta$. This starts the induction.

The inductive step. We now suppose that (3) and (4) hold after step $k *$ (when $\gamma_{i-1}$ was chosen), and verify that $\gamma_{i}$ can be chosen so they hold after step $k+$. We may suppose that $\gamma_{i}$ is to be chosen to equal $\gamma_{i-1} f^{(k+)}$. Recall the simplification of (3) before the inductive step, and the choice of $v$. In particular, after step $k *, f v$ potentially takes $\gamma_{i-1}$ to $\gamma_{l b}$.

First note that (3) does not conflict with a condition of type (1). Suppose $\mu, \lambda \in \Omega$ with $\mu v^{(k *)}=\lambda$, and that $\lambda \sim \gamma_{l b}$. We must choose $\gamma_{i}$ so $\mu \sim \gamma_{i}$. If this clashes with (1), then $\mu\left(f^{-1}\right)^{(k *)} \not \gamma_{i-1}$. However, $f v$ potentially takes (at step $k *$ ) $\gamma_{i-1}$ to $\gamma_{l b}$ and $\left(\mu\left(f^{-1}\right)^{(k *)}\right)(f v)^{(k *)}=\lambda$. Thus, $\lambda \sim \gamma_{l b}$ and $\mu\left(f^{-1}\right)^{(k *)} \not \gamma_{i-1}$, contrary to (3) at the previous substep. We should also consider here the case $\lambda=\gamma_{i}$, in which case we must ensure $\mu \sim \gamma_{i} \leftrightarrow \gamma_{i} \sim \gamma_{l b}$. Again, this is consistent.

Thus, it remains to show that (4) is preserved, under the assumption that (1)-(3) hold after step $k+$. So suppose $\gamma_{i}$ is chosen so that (1)-(3) hold but (4) does not hold, and that the word $z$ is a counterexample to (4) of minimal length. By this minimality we may suppose that in (4), $\alpha=\gamma_{i}$, and that the word $z$ has form $v y_{1} x_{2} y_{2} \cdots x_{r} y_{r}$, where $x_{2}, \ldots, x_{r} \in\left\{v, \dot{v}^{-1}\right\}$, and the $y_{i}$ are arbitrary (reduced) words. Here, after 
step $k *$, each $y_{i}$ is defined on the potential image of $\gamma_{i}$ under $v x_{2} y_{2} \cdots x_{i}$, and the decomposition of $z$ is chosen so that $\alpha v y_{1} \ldots y_{j}$ is potentially $\alpha$ if $x_{j+1}=v$, or $\gamma_{l b}$ if $x_{j+1}=v^{-1}$.

We suppose for a contradiction that $r>1$. By minimality of $\ell(z)$ and the assumption that (1)-(3) hold, $v^{(k+)}$ and $\left(v y_{1}\right)^{(k+)}$ are not potentially defined on $\alpha^{\prime}$ (so eventually, i.e., after step $k$, they will take $\alpha^{\prime}$ to points in $\left.\left\{\gamma_{i+1}, \ldots, \gamma_{l b-1}\right\}\right)$. Also, as $\gamma_{l b} y_{1}^{(k *)} \in\left\{\gamma_{i}, \gamma_{l b}\right\}$ and any word at step $k *$ of length at most $l^{2}$ defined on $\gamma_{l b}$ is an initial subword of $w^{l}, \ell\left(y_{1}\right) \geq l^{2}$, and $y_{1}$ has an initial subword $w$. It follows that after step $k, w$ will be defined on $\alpha^{\prime} v^{(k)}$. In particular, as eventually we will have $\alpha^{\prime} v w \in\left\{\gamma_{i+1}, \ldots, \gamma_{l b}\right\}$ we have $\ell(v)>\ell(w)$, and we can write $v=v^{\prime} w^{q}$ where $\ell\left(v^{\prime}\right)<\ell(w)$ and $q>0$. By Lemma 4.4 (ii), after step $k \alpha^{\prime} v$ will lie on the $w$-cycle of $\gamma_{0}$. Assume first $\ell\left(v^{\prime}\right)>0$. Then as $v^{\prime}$ is a proper final subword of $w$ (and the maximal $w^{(k *)}$-chain of $\gamma_{0}$ begins with a point on the $w$-cycle of $\left.\gamma_{0}\right)$, it follows that $\alpha^{\prime \prime}:=\alpha^{\prime} f^{-1}$ is defined. Hence, after step $k * f z$ potentially takes $\left\langle\gamma_{i-1}, \alpha^{\prime \prime}\right\rangle$ to $\left\langle\beta, \beta^{\prime}\right\rangle$. Hence $\gamma_{i-1} \sim \alpha^{\prime \prime} \leftrightarrow \beta \sim \beta^{\prime}$, so as $f$ is an automorphism, $\gamma_{i} \sim \alpha^{\prime} \leftrightarrow \beta \sim \beta^{\prime}$, as required. If $v^{\prime}$ is empty, so $v=w^{q}$, then, by considering Lemma 4.4 (ii) applied to $\alpha^{\prime} v, \alpha^{\prime} v y_{1}, \alpha^{\prime} v y_{1} x_{2}$, we get that $y_{1}$ is a positive power of $w$. This contradicts that

$$
\gamma_{l b} y_{1}^{(k+)} \in\left\{\gamma_{i}, \gamma_{l b}\right\}
$$

Thus, we reduce to the case $r=1$, that is, $z=v y$. Again, by minimality of $\ell(z)$ we may suppose that after step $k$, each point on the $y^{(k)}$-chain from $\alpha^{\prime} v^{(k)}$ to $\alpha^{\prime}(v y)^{(k)}$ will lie in $\left\{\gamma_{i+1}, \ldots, \gamma_{l b-1}\right\}$, except for $\alpha^{\prime}(v y)^{(k)}$ which is one of $\gamma_{i}, \ldots, \gamma_{l b}$. In particular, $\ell(y)<\ell(v)$. Write $v=v^{\prime} w^{q}$ where $\ell\left(v^{\prime}\right)<l$.

Suppose first $\ell(y) \geq \ell(w)$. Then as $\gamma_{l b} y^{(k *)}$ is defined, $y$ has $w$ as an initial segment. Hence, by Lemma 4.4 (ii), as $y^{(k)}$ will be defined on $\alpha^{\prime} v^{(k)}, \alpha^{\prime} v^{(k)}$ will be on the $w$-cycle of $\gamma_{0}$. In particular, $y$ is a power of $w$, and $\beta^{\prime}=\gamma_{l b}$. In this case, $v y$ is a final segment of a power of $w$, and it potentially takes $\alpha$ to $\beta$ and $\alpha^{\prime}$ to $\beta^{\prime}$ along a subset of the $w$-chain from $\gamma_{-r^{\prime}-1}$ to $\gamma_{l b+s^{\prime}-1}$. We may suppose $v^{\prime}$ is non-empty, as otherwise $z$ is a power of $w$ and hence satisfies (4) by Definition 4.1 (i). In particular, as above $\alpha^{\prime \prime}:=\alpha^{\prime} f^{-1}$ is defined at step $k *$, and $f v y$ potentially takes $\left\langle\gamma_{i-1}, \alpha^{\prime \prime}\right\rangle$ to $\left\langle\beta, \beta^{\prime}\right\rangle$. Since (4) held at step $k *$ and $f$ is a partial automorphism, (4) holds after step $k+$.

Alternatively, $\ell(y)<\ell(w)$. There are two cases, according to whether $\beta^{\prime}$ is $\gamma_{l b}$ or $\gamma_{i}$. In the first case, we have to choose $\gamma_{i}$ to ensure $\gamma_{i} \sim \alpha^{\prime} \leftrightarrow \beta \sim \gamma_{l b}$, and in the second case we choose $\gamma_{i}$ so that $\gamma_{i} \sim \alpha^{\prime} \leftrightarrow \beta \sim \gamma_{i}$. It can be checked that this does not conflict with other constraints.

The above argument shows that the two cycles of $w^{(k-1)}$ can be joined, without creating new incompatible cycles for $w_{0}, \ldots, w_{n-1}$. It remains to check that after Step $5 n+2$, there are no coincidences. By the proof of Lemma 4.3, any such coincidence must involve one of the $\delta_{i}$ and one of the $\delta_{i}^{\prime}$ and for some $e \leq n-1$ must 
involve a $w_{e}$-chain from $\delta_{i}$ to $\delta_{j}^{\prime}$ along $\gamma_{0}, \ldots, \gamma_{l b}$ (we do not mean that the $\delta_{i}, \delta_{i}^{\prime}$ are endpoints of the chains in the coincidence). Furthermore, by Lemma 4.4 (i), $w_{e}=w$.

So suppose we have a $\left(w, w_{e^{\prime}}, k, \alpha, \beta\right)$-coincidence. Since the 'next' letter of the $w$-chain from $\alpha$ to $\beta$ is undefined, we must have $\beta=\alpha_{s^{\prime}}^{\prime}$. However, in this case, some chain of length at least $l^{2}+l$ is both a $w$-chain and a $w_{e^{\prime}}$-chain, so by Lemma 4.4 (i), $w=w_{e}$, and the two chains between $\alpha$ and $\beta$ are equal, contrary to the definition of coincidence.

Step $k=5 n+4$. Put $l:=\max \left\{l_{0}, \ldots, l_{n}\right\}$. Consider all pairs $\langle\epsilon, w\rangle$, where $\epsilon$ is an old point and $w$ is a reduced word of length $l^{2}+l$, whose first letter $h$ does not have $\epsilon \in \operatorname{dom}\left(h^{(k-1)}\right)$. For each such pair, use Lemma 4.3 to add a $w^{(k)}$-chain of length $l^{2}+l$, so that $\epsilon w^{(k)}$ is defined. We do this by a good extension, in such a way that there are no overlaps between the added points for $\langle\epsilon, w\rangle$ and for any other $\left\langle\epsilon^{\prime}, w^{\prime}\right\rangle$, except those forced because $\epsilon=\epsilon^{\prime}$ and $w, w^{\prime}$ have a common initial subword. It follows from Lemma 4.4 that after this step there is no $\left(w_{a}, w_{b}, k, \alpha, \beta\right)$ coincidence for any $a, b \leq n$.

\section{References}

[1] P. J. Cameron, Oligomorphic permutation groups, London Math. Soc. Lecture Notes 152 (Cambridge University Press, Cambridge, 1990).

[2] - 'Cofinitary permutation groups', Bull. London Math. Soc. 28 (1996), 113-140.

[3] J. D. Dixon, 'Most finitely generated permutation groups are free', Bull. London Math. Soc. 22 (1990), 222-226.

[4] D. G. Higman, 'Intersection matrices for finite permutation groups', J. Algebra 6 (1967), 22-42.

[5] G. Karolyi, S. J. Kovacs and P. P. Palfy, 'Doubly transitive permutation groups with abelian stabilisers', Aequationes Math. 39 (1990), 161-166.

[6] A. Kepert and G. A. Willis, 'Scale functions and tree ends', J. Austral. Math. Soc. 70 (2001), 273-292.

[7] H. D. Macpherson, 'Permutation groups whose subgroups have just finitely many orbits', in: Ordered groups and infinite permutation groups (ed. W. C. Holland) (Kluwer, 1996) pp. 221-231

[8] V. D. Mazurov, 'On doubly transitive permutation groups', Sibirsk. Mat. Zh. 31 (1990), 102-104.

[9] G. A. Willis, 'The structure of totally disconnected locally compact groups', Math. Ann. 300 (1994), 341-363.

[10] — 'Totally disconnected nilpotent locally compact groups', Bull. Austral. Math. Soc. 55 (1997), 143-146.

Department of Mathematics

Indian Institute of Technology Guwahati

Guwahati

Assam 781039

India
Department of Pure Mathematics

University of Leeds

Leeds LS2 9JT

England

e-mail: h.d.macpherson@leeds.ac.uk

e-mail: meenaxi@iitg.ernet.in 
